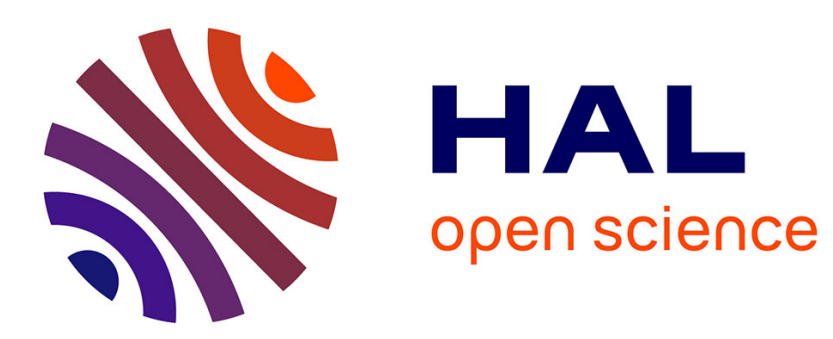

\title{
Relaxed persistent Flow/Jump Conditions for Uniform Global Asymptotic Stability
}

Christophe Prieur, Andrew Teel, Luca Zaccarian

\section{To cite this version:}

Christophe Prieur, Andrew Teel, Luca Zaccarian. Relaxed persistent Flow/Jump Conditions for Uniform Global Asymptotic Stability. IEEE Transactions on Automatic Control, 2014, 59 (10), pp.27662771. 10.1109/TAC.2014.2311611 . hal-01066807

\section{HAL Id: hal-01066807 https://hal.science/hal-01066807}

Submitted on 22 Sep 2014

HAL is a multi-disciplinary open access archive for the deposit and dissemination of scientific research documents, whether they are published or not. The documents may come from teaching and research institutions in France or abroad, or from public or private research centers.
L'archive ouverte pluridisciplinaire HAL, est destinée au dépôt et à la diffusion de documents scientifiques de niveau recherche, publiés ou non, émanant des établissements d'enseignement et de recherche français ou étrangers, des laboratoires publics ou privés. 


\section{Relaxed persistent flow/jump conditions for uniform global asymptotic stability}

Christophe Prieur, ${ }^{1}$ Andrew R. Teel, ${ }^{2}$ Luca Zaccarian ${ }^{3}$

\begin{abstract}
For hybrid systems, sufficient conditions are derived for the uniform global asymptotic stability of a given closed set. These conditions are written in terms of a Lyapunov function candidate and assume a semiglobal practical persistent flow (resp. persistent jump) property of the solutions to the hybrid system. The use of the new conditions is illustrated via the stability analysis of a physically inspired example and of an eventtriggered control algorithm.
\end{abstract}

Index Terms-hybrid dynamical systems, Lyapunov methods, uniform global asymptotic stability, event-triggered control

\section{INTRODUCTION}

$\mathbf{H}$ YBRID dynamical systems can characterize continuous evolution (or flow) and discrete evolution (or jump) of their solutions. Examples of hybrid dynamical systems include, e.g., systems having an internal clock (such as the flashing fireflies), or mechanical objects experiencing mixed discrete and continuous dynamics (such as the bouncing ball). (See [4, Chap. 1] for more examples.) While many mathematical frameworks have been proposed to suitably represent hybrid dynamics (see, e.g., the ones cited in [5]), a suggestive one is that recently surveyed in [4], [5] which is capable of covering the well understood continuous- and discrete-time dynamical systems as special cases.

In [4, Chap. 3] it is proven that if a Lyapunov function candidate strictly decreases along flows and across jumps, then the attractor is uniformly globally pre-asymptotically stable (UGpAS). Some relaxed conditions are then given in $[4, \mathrm{Sec}$. 3.3] where it is shown that one may relax the strict decrease across jumps (respectively along flows) if a suitable persistent flow (respectively persistent jump) condition is satisfied by the solutions. The aim of this paper is to show that a relaxed semiglobal practical notion of persistent flow/jump is still sufficient to establish UGpAS. Our main results, given in Section II, use a lemma stating that uniform global stability (UGS) plus semiglobal practical uniform global pre-attractivity implies UGpAS. This lemma is related to [14, Theorem 1] for continuous-time systems.

A possible use of the proposed UGpAS conditions is illustrated in Section III-A where we use our practical persistent

${ }^{1}$ Christophe Prieur is with Gipsa-lab, Grenoble Campus, 11 rue des Mathématiques, BP 46, 38402 Saint Martin d'Hères Cedex, France, christophe.prieur@gipsa-lab.fr.

${ }^{2}$ Andrew R. Teel is with ECE Department, University of California, Santa Barbara (CA), USA, teel@ece. ucsb. edu. Research supported in part by AFOSR grant number FA9550-12-1-0127 and NSF grant number ECCS1232035 .

${ }^{3}$ Luca Zaccarian is with CNRS; LAAS; 7 avenue du Colonel Roche F31077 and Université de Toulouse; UPS, INSA, INP, ISAE, UT1, UTM, LAAS; F-31077, Toulouse, France, and with Dip. di Ingegneria Industriale, University of Trento, Italy, zaccarian@laas. fr. The work of CP and LZ is supported by the ANR project LimICoS contract number 12 BS03 00501 , and by HYCON2 Network of Excellence "Highly-Complex and Networked Control Systems", grant agreement 257462. jump property to establish UGpAS for a nonlinear mass-spring system with impacts having a (non-necessarily periodic) time varying restitution coefficient. For this time-varying system the attractor is closed but not bounded, therefore the La Salle's results of [9] cannot be applied (whereas one may use the Matrosov constructions in [10]). A second example, treated in Section III-B, illustrates the use of practical persistent flow by providing a more elegant proof of the main result in [11], in the context of event-triggered controllers (see, e.g., [2], [3], [11], [12]). The algorithm of [11] satisfies our semiglobal practical persistent flow condition (but not the persistent flow condition of [4, Sec. 3.3]), and thus we can apply the results of this paper to prove UGpAS of the attractor. This result generalizes [11] since uniformity of GAS could not be proven with the approach adopted in [11]. Moreover, we require milder regularity conditions than [11] and prove a robustness result, stated in Section V. This last result strengthens the result in [1, Claim 5.1] and is of interest on its own.

Notation: A function $\alpha:[0, \infty) \rightarrow \mathbb{R}$ is of class $\mathcal{K}$ if it is zero at zero, continuous and strictly increasing. It is of class $\mathcal{K}_{\infty}$ if it is of class $\mathcal{K}$ and is unbounded. A function $\rho:[0, \infty) \rightarrow \mathbb{R}$ belongs to $\mathcal{P} \mathcal{D}$ (positive definite) if it is continuous, $\rho(s)>0$ for all $s>0$ and $\rho(0)=0$. Given a set $C \subset \mathbb{R}^{n}, \bar{C}$ denotes its closure. Given a set $\mathcal{A} \subset \mathbb{R}^{n}$, and a point $x \in \mathbb{R}^{n},|x|_{\mathcal{A}}:=\inf _{z \in \mathcal{A}}|x-z|$. For any integer $m$ and any scalar $\delta>0$, the set $\delta \mathbb{B}^{\circ}:=\left\{x \in \mathbb{R}^{m}:|x|<\delta\right\}$ denotes the (open) $\delta$-ball centered at the origin and $\delta \mathbb{B}$ denotes its closure. Given two sets $\mathcal{X}, \mathcal{Y} \subset \mathbb{R}^{n}$, the set $\mathcal{X}+\mathcal{Y}$ comprises all vectors $z=x+y$ for some $x \in \mathcal{X}$ and $y \in \mathcal{Y}$.

\section{MAIN RESUlTS}

Consider the following nonlinear hybrid dynamical system $\mathcal{H}=(C, F, D, G)$ :

$$
\mathcal{H}\left\{\begin{aligned}
\dot{x} & \in F(x), & & x \in C \\
x^{+} & \in G(x), & & x \in D
\end{aligned}\right.
$$

where $F$ and $G: \mathbb{R}^{n} \rightrightarrows \mathbb{R}^{n}$ are locally bounded set-valued mappings and $C$ and $D$ are subsets of $\mathbb{R}^{n}$. Let $\mathcal{A}$ be a closed subset of $\mathbb{R}^{n}$. For an introduction to hybrid systems notation and precise definitions of solutions to (1) and hybrid time domains, the reader is referred to [4, $\$ 2.2-2.3]$. We recall here the following from [4, Definition 3.6]:

Definition 1: (Uniform global stability concepts)

- The set $\mathcal{A}$ is uniformly globally stable (UGS) for (1) if there exists a class $\mathcal{K}_{\infty}$ function $\alpha$ such that any solution $x$ to (1) satisfies $|x(t, j)|_{\mathcal{A}} \leq \alpha\left(|x(0,0)|_{\mathcal{A}}\right)$, for all $(t, j) \in \operatorname{dom} x$;

- the set $\mathcal{A}$ is uniformly globally pre-attractive (UGpA) for (1) if for each $\varepsilon>0$ and $r>0$, there exists $T>0$ such that for any solution $x$ to (1),

$$
\begin{aligned}
|x(0,0)|_{\mathcal{A}} \leq r \Rightarrow & |x(t, j)|_{\mathcal{A}} \leq \varepsilon, \\
& \forall(t, j) \in \operatorname{dom} x, t+j \geq T .
\end{aligned}
$$

- the set $\mathcal{A}$ is uniformly globally pre-asymptotically stable (UGpAS) for (1) if it is both uniformly globally stable and uniformly globally pre-attractive.

- the set $\mathcal{A}$ is uniformly globally asymptotically stable (UGAS) if it is uniformly globally pre-asymptotically stable and all maximal solutions to (1) are complete. 
Note that once UGS of $\mathcal{A}$ for $\mathcal{H}$ is established, the remaining UGpA property amounts to checking uniform convergence in a semiglobal practical way (that is, for each pair $r>0, \varepsilon>0$ ). Then we can build a set of relaxed conditions ensuring UGpA of $\mathcal{A}$ by introducing, for each pair $0<\delta<\Delta$ of positive scalars, the following set

$$
\mathcal{S}_{\delta, \Delta}:=(\mathcal{A}+\Delta \mathbb{B}) \backslash\left(\mathcal{A}+\delta \mathbb{B}^{\circ}\right),
$$

(which is closed, not necessarily bounded, and is bounded if and only if $\mathcal{A}$ is bounded) and introducing the following hybrid system

$$
\begin{aligned}
\mathcal{H}_{\delta, \Delta} & =\left(C_{\delta, \Delta}, F, D_{\delta, \Delta}, G\right) \\
& :=\left(C \cap \mathcal{S}_{\delta, \Delta}, F, D \cap \mathcal{S}_{\delta, \Delta}, G\right),
\end{aligned}
$$

which corresponds to restricting the flow and jump sets to the closed set $\mathcal{S}_{\delta, \Delta}$.

In $[4, \S 3.2]$, Lyapunov-based sufficient conditions for UGAS of a closed set $\mathcal{A}$ are given in terms of the so-called "strict" Lyapunov conditions, namely strict decrease of a Lyapunov function both across jumps and along flows. Then, in [4, §3.3], several relaxations of the strict Lyapunov conditions are given, among which we focus here on the ones called "persistent jumping" [4, Prop. 3.24] and "persistent flowing" [4, Prop. 3.27]. In light of the restriction in (3), we introduce a relaxed semiglobal practical formulation of persistent jump/flow.

Property 1: (Semiglobal practical persistent flow [respectively, persistent jump]) Given system (1), for each pair $\delta, \Delta$ of positive scalars, there exist a class $\mathcal{K}_{\infty}$ function $\gamma$ and a scalar $N \geq 0$ such that each solution $x$ to hybrid system $\mathcal{H}_{\delta, \Delta}$ in (3) satisfies the following for all $(t, j) \in \operatorname{dom} x$ :

$$
\begin{array}{r}
t \geq \gamma(j)-N, \\
\text { [respectively, } j \geq \gamma(t)-N] .
\end{array}
$$

Semiglobal practical persistent flow [respectively, jump] is useful because it still allows to assess UGpAS of an attractor when strict decrease of a candidate Lyapunov function is only established along flows [respectively, across jumps] and only non-increase of the Lyapunov function is established across jumps [respectively, along flows] (see [4, Props. 3.27 and 3.24]). In other words these properties allow establishing UGpAS in the presence of nonstrict (weakened) Lyapunov conditions. The following main results of this paper, generalize [4, Props. 3.27 and 3.24]. They are proven in Section IV.

Theorem 1: Consider hybrid system $\mathcal{H}=(C, F, D, G)$ in (1) and a closed set $\mathcal{A} \subset \mathbb{R}^{n}$. Assume that there exist a function $V$, continuously differentiable on a neighborhood of $\bar{C}$, two class $\mathcal{K}_{\infty}$ functions $\alpha_{1}$ and $\alpha_{2}$, and a function $\rho \in \mathcal{P} \mathcal{D}$ such that

$$
\begin{array}{ll}
\alpha_{1}\left(|x|_{\mathcal{A}}\right) \leq V(x) \leq \alpha_{2}\left(|x|_{\mathcal{A}}\right), & \forall x \in C \cup D \cup G(D), \\
\langle\nabla V(x), f\rangle \leq-\rho\left(|x|_{\mathcal{A}}\right), & \forall x \in C, \forall f \in F(x), \\
V(g)-V(x) \leq 0, & \forall x \in D, \forall g \in G(x) .
\end{array}
$$

Assume also that the semiglobal practical persistent flow Property 1 holds for (1). Then the set $\mathcal{A}$ is uniformly globally pre-asymptotically stable for system (1).

Theorem 2: Consider hybrid system $\mathcal{H}=(C, F, D, G)$ in (1) and a closed set $\mathcal{A} \subset \mathbb{R}^{n}$. Assume that there exist a function $V$, continuously differentiable on a neighborhood of $\bar{C}$, two class $\mathcal{K}_{\infty}$ functions $\alpha_{1}$ and $\alpha_{2}$, and a function $\rho \in \mathcal{P} \mathcal{D}$ satisfying (6a) and

$$
\begin{array}{ll}
\langle\nabla V(x), f\rangle \leq 0, & \forall x \in C, \forall f \in F(x), \\
V(g)-V(x) \leq-\rho\left(|x|_{\mathcal{A}}\right), & \forall x \in D, \forall g \in G(x) .
\end{array}
$$

Assume also that the semiglobal practical persistent jump Property 1 holds for (1). Then the set $\mathcal{A}$ is uniformly globally pre-asymptotically stable for system (1).

Theorems 1 and 2 differ in a few ways from the results in [4, Props. 3.27 and 3.24] as clarified next.

- First, using the restriction in (3), we are allowed to disregard a neighborhood of the attractor $\mathcal{A}$ for the persistent flow/jump. This is useful to rule out defective solutions only occurring inside the attractor. Examples of such cases correspond to the situations addressed in Sections III-A and III-B, the reset rules proposed in [8], the bouncing ball example (see [4, Example 3.19]) or the homogeneous approximations in [7] where the attractor $\mathcal{A}=\{0\}$ necessarily belongs to both the jump and flow sets which are closed cones.

- In [4, Props. 3.27 and 3.24], the argument of the class $\mathcal{K}_{\infty}$ function $\gamma$ in (4) [respectively, (5)] is $t+j$. The new formulation in Property 1 seems to be easier to establish, due to the fact that the variable $t$ [respectively, $j$ ] appearing at the left hand side does not appear at the right hand side. It certainly leads to an easier proof of Propositions 1 and 2 in Section III. The next lemma shows that the two formulations are equivalent.

Lemma 1: Given a hybrid time domain $E$, there exist $\gamma \in \mathcal{K}_{\infty}$ and $N \geq 0$ satisfying (4) [respectively, (5)] for all $(t, j) \in E$, if and only if there exist $\hat{\gamma} \in \mathcal{K}_{\infty}$ and $\hat{N} \geq 0$ satisfying, for all $(t, j) \in E$ :

$$
\begin{aligned}
t & \geq \hat{\gamma}(t+j)-\hat{N}, \\
\text { [respectively, } & \geq \hat{\gamma}(t+j)-\hat{N}] .
\end{aligned}
$$

Proof. We only prove the case corresponding to (4), (8). The other proof is identical with $t$ and $j$ exchanged. If (8) holds for some $\hat{\gamma} \in \mathcal{K}_{\infty}$ and $\hat{N} \geq 0$, then it suffices to pick $\gamma=\hat{\gamma}$ and $N=\hat{N}$ for (4) to hold, because $t \geq 0$ and $\hat{\gamma} \in \mathcal{K}_{\infty}$ implies $t \geq \hat{\gamma}(t+j)-\hat{N} \geq \hat{\gamma}(j)-\hat{N}$. Conversely, assume that (4) holds for some $\gamma \in \mathcal{K}_{\infty}$ and $N \geq 0$. Then the choice $\hat{N}=\frac{N}{2}$ and $\hat{\gamma}(s)=\frac{1}{2} \gamma_{m}\left(\frac{s}{2}\right)$ with $\gamma_{m}(s)=\min \{s, \gamma(s)\}$ satisfies (8). To see this, add $t$ to both sides of (4) to get $2 t \geq t+\gamma(j)-N$ which implies

$$
\begin{aligned}
t & \geq \frac{1}{2}(t+\gamma(j))-\frac{N}{2} \\
& \geq \frac{1}{2}\left(\gamma_{m}(t)+\gamma_{m}(j)\right)-\hat{N} \\
& \geq \frac{1}{2} \gamma_{m}\left(\frac{t+j}{2}\right)-\hat{N}=\hat{\gamma}(t+j)-\hat{N}
\end{aligned}
$$

where the last inequality follows from inspecting the following two cases: (i) $t \geq j$ implies $\gamma_{m}(t)+\gamma_{m}(j) \geq \gamma_{m}(t)=$ $\gamma_{m}\left(\frac{t+t}{2}\right) \geq \gamma_{m}\left(\frac{t+j}{2}\right)$ and (ii) $j \geq t$ implies $\gamma_{m}(t)+\gamma_{m}(j) \geq$ $\gamma_{m}(j)=\gamma_{m}\left(\frac{j+j}{2}\right) \geq \gamma_{m}\left(\frac{t+j}{2}\right)$.

\section{ILLUSTRATIONS}

\section{A. Nonlinear impacting mass-spring system}

Let us consider a mass connected to a nonlinear spring. According to the sketch to the top left of Figure 1, the mass 
is moving along the horizontal axis with a position $p \geq 0$ and a velocity $v=\dot{p}$. Assume moreover that the mass is impacting with a vertical wall at $p=0$, where each dissipative damping $h \in \mathbb{Z}$ is associated to the restitution coefficient $0 \leq \Gamma(h) \leq$ $\Gamma_{0}<1$. This gives the following hybrid model which uses the counter $h \in \mathbb{Z}$ and the state $x=(p, v, h)$ :

$$
\begin{aligned}
& {\left[\begin{array}{l}
\dot{p} \\
\dot{v} \\
\dot{h}
\end{array}\right]=\left[\begin{array}{c}
v \\
-k(p) \\
0
\end{array}\right], \quad x \in C:=\left\{x \in \mathbb{R}^{2} \times \mathbb{Z}, \text { s.t. } p \geq 0\right\}} \\
& {\left[\begin{array}{c}
p^{+} \\
v^{+} \\
h^{+}
\end{array}\right]=\left[\begin{array}{c}
0 \\
-\Gamma(h) v \\
h+1
\end{array}\right], \quad x \in D:=\left\{x \in \mathbb{R}^{2} \times \mathbb{Z},\right.} \\
& \text { s.t. } p=0 \text { and } v \leq 0\},
\end{aligned}
$$

and that satisfies the following assumption characterizing a very general nonlinear spring with the only requirements that at rest $(p=0)$ the spring exerts no force and that extending the spring one experiences an increase of elastic force.

Assumption 1: Function $k$ is of class $\mathcal{K}$ and ${ }^{4}$ there exists a scalar $\Gamma_{0} \in[0,1)$ such that $0 \leq \Gamma(h) \leq \Gamma_{0}$ for all $h \in \mathbb{Z}$.
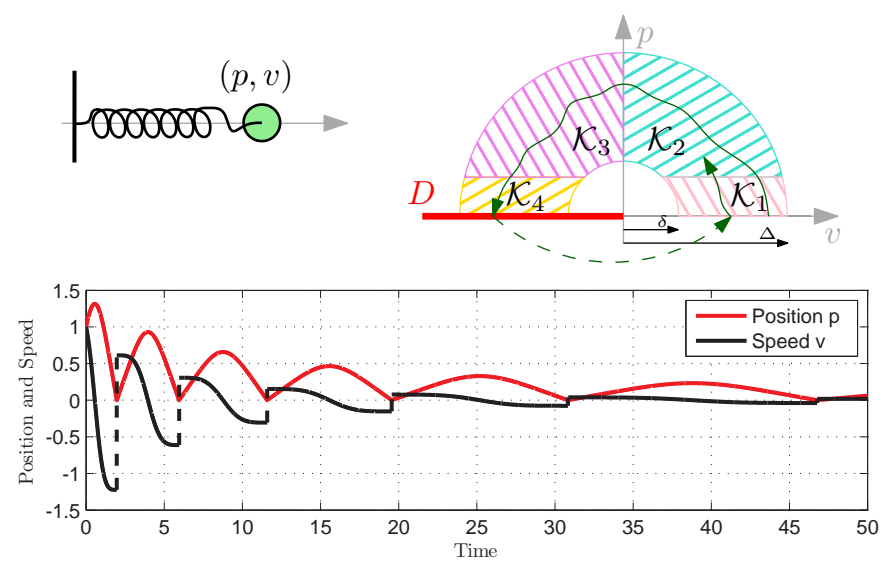

Fig. 1. Sketch of the mass-spring system of Section III-A, its flow and jump sets, and a solution starting from $x(0,0)=(1,1)$ projected on the ordinary time domain $t$.

From the simulation of Figure 1, corresponding to the selection $k(p)=p^{3}$ and $\Gamma(h)=\frac{1}{2}, \forall h$, one gets a clear impression that the attractor

$$
\mathcal{A}:=\left\{(p, v, h) \in \mathbb{R}^{2} \times \mathbb{Z}:(p, v)=(0,0)\right\}
$$

is globally asymptotically stable for the dynamics. Nevertheless, formally proving this fact may be complicated because no dissipation happens during flow and the impacts, which dissipate, may happen increasingly seldom as the mass approaches zero, especially for specific defective shapes of the very general function $k$. Nevertheless, we may prove UGAS of the origin by exploiting Theorem 2 as formally stated next.

Proposition 1: Under Assumption 1, the closed set $\mathcal{A}$ in (11) is UGAS for system (10).

Proof. The proof uses Theorem 2 with $F$ and $G$ being the two right hand sides in (10), $C$ and $D$ as defined in (10) and the closed attractor $\mathcal{A}$ in (11). To construct the Lyapunov

\footnotetext{
${ }^{4}$ It is actually enough that $k$ be positive definite and not integrable, so that the Lyapunov function in the proof of Proposition 1 is radially unbounded.
}

function $V$, we consider the energy of the system during flow, that is $V(x):=\int_{0}^{p} k(s) d s+\frac{1}{2} v^{2}$. For this $V$, after noticing that $|x|_{\mathcal{A}}=|(p, v)|$, we may establish (6a) as follows:

$\min \left\{\int_{0}^{\frac{|x|_{\mathcal{A}}}{\sqrt{2}}} k(s) d s, \frac{1}{4}|x|_{\mathcal{A}}^{2}\right\} \leq V(x) \leq \int_{0}^{|x|_{\mathcal{A}}} k(s) d s+\frac{1}{2}|x|_{\mathcal{A}}^{2}$,

indeed the right inequality is easily checked by writing an upper bound for each term of $V$ and the left inequality can be checked by first noticing that in $C \cup D \cup G(D)$ one always has $p=|p|$ and then splitting the analysis in two cases: 1) if $|p| \geq|v|$, then $|p| \geq \frac{|x|_{\mathcal{A}}}{\sqrt{2}}$ and thus $V(x) \geq \int_{0}^{\frac{|x|_{\mathcal{A}}}{\sqrt{2}}} k(s) d s$ and 2) if $|v| \geq|p|$, then $|v| \geq \frac{|x|_{\mathcal{A}}}{\sqrt{2}}$ and thus $V(x) \geq \frac{1}{4}|x|_{\mathcal{A}}^{2}$. Let us now show equations (7). The flow inequality (7a) is easily established by noticing that, for all $x \in C,\left\langle\nabla V(x),\left[\begin{array}{c}v \\ -k(p) \\ 0\end{array}\right]\right\rangle=0$. The jump inequality (7b) is established with $\rho(s)=\left(1-\Gamma_{0}\right)^{2} s^{2}$, indeed for all $x \in D$ we have $V\left(\left[\begin{array}{c}0 \\ -\Gamma(h) v \\ h+1\end{array}\right]\right)-V(x)=-\left(1-\Gamma(h)^{2}\right)|v|^{2}=-\rho\left(|x|_{\mathcal{A}}\right)$.

The last thing to establish to apply Theorem 2 is the semiglobal practical persistent jump Property 1 . To this aim, for each pair of positive scalars $0<\delta<\Delta$, consider system $\mathcal{H}_{\delta, \Delta}$ defined in (3) and notice that any solution to this system can flow for a uniformly bounded ordinary time interval. Indeed, each solution to $\mathcal{H}_{\delta, \Delta}$ experiencing flow is guaranteed to exit from the flow set (the compact set $\mathcal{K}_{\delta, \Delta}$ below corresponds to the tiled areas at the right of Figure 1):

$$
C_{\delta, \Delta}=\mathcal{K}_{\delta, \Delta} \times \mathbb{Z}=(\{\delta \leq|(p, v)| \leq \Delta\} \cap\{p \geq 0\}) \times \mathbb{Z}
$$

after a bounded time. This simple observation comes from the circulant nature of the trajectories but can be more formally proven by focusing on the sets $\mathcal{K}_{1}-\mathcal{K}_{4}$ represented in Figure 1 , $\mathcal{K}_{1}:=\mathcal{K}_{\delta, \Delta} \cap\{p \leq \delta / \sqrt{2}$ and $v \geq 0\}, \mathcal{K}_{2}:=\mathcal{K}_{\delta, \Delta} \cap\{p \geq$ $\delta / \sqrt{2}$ and $v \geq 0\}, \mathcal{K}_{3}:=\mathcal{K}_{\delta, \Delta} \cap\{p \geq \delta / \sqrt{2}$ and $v \leq 0\}$, and $\mathcal{K}_{4}=\mathcal{K}_{\delta, \Delta} \cap\{p \leq \delta / \sqrt{2}$ and $v \leq 0\}$. In particular, notice that the $(p, v)$ components of all flowing solutions

1. must leave after a finite time the set $\mathcal{K}_{1}$ and enter $\mathcal{K}_{2}$ or exit $\mathcal{K}_{\delta, \Delta}$ because in $\mathcal{K}_{1}$ we have $\dot{p}=v \geq \frac{\delta}{\sqrt{2}}$;

2. must leave after a finite time the set $\mathcal{K}_{2}$ and enter $\mathcal{K}_{3}$ or exit $\mathcal{K}_{\delta, \Delta}$ because in $\mathcal{K}_{2}$ we have $\dot{p}=v \geq 0$ and $\dot{v}=-k(p) \leq-k\left(\frac{\delta}{\sqrt{2}}\right)$

3. must leave after a finite time the set $\mathcal{K}_{3}$ and enter $\mathcal{K}_{4}$ or exit $\mathcal{K}_{\delta, \Delta}$ because in $\mathcal{K}_{3}$ we have $\dot{v}=-k(p) \leq-k\left(\frac{\delta}{\sqrt{2}}\right)$

4. must leave after a finite time the set $\mathcal{K}_{4}$ and exit $\mathcal{K}_{\delta, \Delta}^{\sqrt{2}}$ because in $\mathcal{K}_{4}$ we have $\dot{p}=v \leq-\frac{\delta}{\sqrt{2}}$.

As a consequence each projection in the $(p, v)$ plane of the solution flowing in the compact set $\mathcal{K}_{\delta, \Delta}$ is guaranteed to exit $\mathcal{K}_{\delta, \Delta}$ in finite (ordinary) time, thereby either having to jump or to terminate. Then, from compactness of $\mathcal{K}_{\delta, \Delta}$ and continuity of (continuous-time) solutions on compact time intervals, in addition to the fact that the flow dynamics is independent of $h$, we obtain that there is a uniform maximum flow time $T_{M}(\delta, \Delta)$ for all solutions to $\mathcal{H}_{\delta, \Delta}$. Finally, equation (5) holds with $\gamma(t)=t / T_{M}(\delta, \Delta)$ and $N=1$. (Note that establishing condition (9) would require additional steps in this proof. This shows that (5) is a simpler condition to check for this example.) 
Since all the assumptions of Theorem 2 hold, then the origin is is UGpAS for (10). To show UGAS, note that from [6, Prop. 2.1], we have local existence of solutions and so the only way a maximal solution would not be complete is if, along flows, it escapes to infinity in finite time. From (7a), the function $V$ is non-increasing along all solutions to (16) and $V$ is radially unbounded in $(p, v)$. Thus finite-time escapes of $V$ are avoided. Moreover $h$ does not escape in finite time because $\dot{h}=0$. Therefore, UGpAS implies UGAS of $\mathcal{A}$.

\section{B. An event-triggered control scheme}

Based on the results in [11], we consider the following nonlinear continuous-time plant with control input $u \in \mathbb{R}^{m}$ :

$$
\dot{x}_{p}=f_{p}\left(x_{p}, u\right)
$$

where $f_{p}: \mathbb{R}^{n_{p}} \times \mathbb{R}^{m} \rightarrow \mathbb{R}^{n_{p}}$ is continuous in both arguments. Paralleling [11], we assume for (12) the existence of a stabilizing state feedback control law $u=\kappa\left(x_{p}\right)$, where $\kappa$ is only required to be locally bounded.

Assumption 2: Function $f_{p}$ is continuous in both arguments and there exist a compact set $\mathcal{A}_{p} \subset \mathbb{R}^{n_{p}}$, a locally bounded function $\kappa: \mathbb{R}^{n_{p}} \rightarrow \mathbb{R}^{m}$, a continuously differentiable function $V_{p}: \mathbb{R}^{n_{p}} \rightarrow \mathbb{R}$, two class $\mathcal{K}_{\infty}$ functions $\alpha_{1}$ and $\alpha_{2}$, and a function $\rho_{\circ} \in \mathcal{P D}$ such that, for all $x_{p} \in \mathbb{R}^{n_{p}}$, the following holds for all $x_{p} \in \mathbb{R}^{n_{p}}$ :

$$
\begin{aligned}
& \alpha_{1}\left(\left|x_{p}\right|_{\mathcal{A}_{p}}\right) \leq V_{p}\left(x_{p}\right) \leq \alpha_{2}\left(\left|x_{p}\right|_{\mathcal{A}_{p}}\right), \\
& \left\langle\nabla V_{p}\left(x_{p}\right), f_{p}\left(x_{p}, \kappa\left(x_{p}\right)\right)\right\rangle \leq-\rho_{\circ}\left(\left|x_{p}\right|_{\mathcal{A}_{p}}\right) .
\end{aligned}
$$

Different from [11], we enforce mild assumptions on the stabilizer $\kappa$. Therefore, we will use its outer semi-continuous regularization (see [4, Lemma 5.16] and references therein):

$$
K\left(x_{p}\right):=\bigcap_{\delta>0} \overline{\kappa\left(x_{p}+\delta \mathbb{B}\right)} .
$$

Then, inspired by [11], we establish robust stability properties of an event-triggered hybrid implementation of the stabilizing law $\kappa$, whose state is $x=\left[\begin{array}{ll}x_{p}^{\top} & u^{\top}\end{array}\right]^{\top}$ and whose (perturbed) dynamics can be written as:

$$
\mathcal{H}: \begin{cases}\dot{x}_{p}=f_{p}\left(x_{p}, u\right), & \left(x_{p}, u\right) \in C \\ \dot{u} \in f_{u}\left(x_{p}\right) \mathbb{B}, & \\ x_{p}^{+}=x_{p} & \left(x_{p}, u\right) \in D, \\ u^{+} \in K\left(x_{p}+\chi\left(x_{p}\right) \mathbb{B}\right)+\chi\left(x_{p}\right) \mathbb{B}, & \end{cases}
$$

where $f_{u}: \mathbb{R}^{n_{p}} \rightarrow \mathbb{R}$ is any continuous function allowing for very general intersample behavior for the plant input $u$, while $\chi: \mathbb{R}^{n_{p}} \rightarrow \mathbb{R}_{\geq 0}$ is a sufficiently small continuous function as specified below. The flow and jump sets are chosen as:

$$
\begin{aligned}
& C=\left\{\left(x_{p}, u\right):\left\langle\nabla V_{p}\left(x_{p}\right), f_{p}\left(x_{p}, u\right)\right\rangle \leq-\rho\left(\left|x_{p}\right|_{\mathcal{A}_{p}}\right)\right\} \\
& D=\left\{\left(x_{p}, u\right):\left\langle\nabla V_{p}\left(x_{p}\right), f_{p}\left(x_{p}, u\right)\right\rangle \geq-\rho\left(\left|x_{p}\right|_{\mathcal{A}_{p}}\right)\right\}
\end{aligned}
$$

and $\rho \in \mathcal{P D}$ is such that $\rho(s)<\rho_{\circ}(s)$ for all $s>0$. This event-triggered algorithm was proposed in [12] and used in many later works. As in [11], we study here the stability properties of the closed (but noncompact) set $\mathcal{A}=\mathcal{A}_{p} \times \mathbb{R}^{m}$ for dynamics (16).
Remark 1: As compared to [11, Thm 3.1], we address here a more general case. In particular, we assume only continuity of $f_{p}$ (while a locally Lipschitz $f_{p}$ was considered there); we allow for a very general flow map for $u$, by way of the continuous function $f_{u}$, while only the case $f_{u}=0$ was considered there; finally we allow for stabilizing laws $\kappa$ satisfying local boundedness only, whereas in [11] continuity of the stabilizer was required. The reason for these relaxations is that not more than those weak properties is required to prove our stability results and the general formulation used here allows to establish robust stability of a larger class of systems. Note however that the special case where $\dot{u}=0$ and $u^{+}=\kappa\left(x_{p}\right)$ is captured by dynamics (16a) by selecting $f_{u}=0$ and noticing that $\kappa\left(x_{p}\right) \in K\left(x_{p}\right)$ for all $x_{p}$. The extra elements of $K\left(x_{p}\right)$ introduced by the regularization (15) are needed to ensure outer semicontinuity of the right hand side of the jump equation which, based on the developments in [4, Chap. 6] ensures well posedness of the hybrid system. This well posedness property enables us to also introduce the perturbation function $\chi$ in the jump equation of (16a) to characterize robustness of UGAS in the presence of small measurement errors (captured by the inner inflation of $K$ ) and round-off errors in the transmission of the signal $u$ (captured by the outer inflation of $K$ ). Note that this robustness result can not be established using [4, Thm 7.21] because the attractor $\mathcal{A}$ is not compact.

Besides the generalizations highlighted in Remark 1, the event-triggered implementation (16) of the stabilizer $\kappa$ has been proven in [11, Thm 3.1] to induce global asymptotic stability (GAS) of $\mathcal{A}=\mathcal{A}_{p} \times \mathbb{R}^{m}$ with $\mathcal{A}_{p}=\{0\}$. However, due to the invariance principle based proof adopted there, appropriately since solutions are bounded, there is no guarantee of the uniformity and robustness of GAS of $\mathcal{A}$. Indeed, since $\mathcal{A}$ is not compact, it is not possible to use the results of [4, Ch. 7]. (See, e.g, [4, Thms $7.12 \&$ 7.21] establishing uniformity and robustness of pre-asymptotic stability with compact attractors.) Instead, using our Theorem 1, it is possible to prove uniformity and robustness of GAS of the attractor by relying on the semiglobal practical persistent flow property established in the proposition below whose proof uses the result of the next claim. The proof of the claim is given in Section V.

Claim 1: Under Assumption 2, letting $n=n_{p}+m$, and $\mathcal{A}=\mathcal{A}_{p} \times \mathbb{R}^{m}$, there exists a small enough function $\chi: \mathbb{R}^{n_{p}} \rightarrow$ $\mathbb{R}_{\geq 0}$ which is positive in $\mathbb{R}^{n_{p}} \backslash \mathcal{A}_{p}$, such that system $\mathcal{H}$ in (16) satisfies the semiglobal practical persistent flow in Property 1.

Remark 2: Note that due to the very mild conditions required by Assumption 2, there is no possibility to prove that there is a minimum intersample behavior guaranteed by the event-triggered implementation of [11, Thm 3.1] (namely a uniform persistent flow property, rather than the practical one established above). Indeed, for very defective nonlinear selections of $f_{p}$ and $\kappa$, one may run into the need of arbitrarily fast sampling close to the origin or at infinity (notice however that for those defective selections no periodic sampling implementation would work either). Nevertheless, if a lower bound on the maximum intersample time is imposed by technological needs, one may still use the robustness properties established here to conclude some kind of semiglobal practical uniform 
asymptotic stability results.

Proposition 2: Under Assumption 2, there exists a small enough function $\chi: \mathbb{R}^{n_{p}} \rightarrow \mathbb{R}_{\geq 0}$ which is positive in $\mathbb{R}^{n_{p}} \backslash \mathcal{A}_{p}$, such that the set $\mathcal{A}=\mathcal{A}_{p} \times \mathbb{R}^{m}$ is UGAS for system (16).

Proof. Introduce the function $V(x)=V\left(\left(x_{p}, u\right)\right)=V_{p}\left(x_{p}\right)$ and note that due to the definition of the set $\mathcal{A}$ we have $\left|\left(x_{p}, u\right)\right|_{\mathcal{A}}=\left|x_{p}\right|_{\mathcal{A}_{p}}$ and from (13), we have

$$
\alpha_{1}\left(|x|_{\mathcal{A}}\right) \leq V(x) \leq \alpha_{2}\left(|x|_{\mathcal{A}}\right) .
$$

Moreover, from the definition of the flow set in (16b), we have

$$
\begin{aligned}
&\left\langle\nabla V(x),\left[\begin{array}{c}
f_{p}\left(x_{p}, u\right) \\
w
\end{array}\right]\right\rangle-\rho\left(|x|_{\mathcal{A}}\right), \\
& \forall\left(x_{p}, u\right) \in C, \forall w \in f_{u}\left(x_{p}\right) \mathbb{B},
\end{aligned}
$$

and since $x_{p}^{+}=x_{p}$, we also have

$$
V\left(x^{+}\right)-V(x)=V_{p}\left(x_{p}^{+}\right)-V_{p}\left(x_{p}\right)=0, \forall\left(x_{p}, u\right) \in D .
$$

Then, by the semiglobal practical persistent flow property established in Claim 1 and by Theorem 1 , set $\mathcal{A}$ is UGpAS for $\mathcal{H}$. To show UGAS, note that from [6, Prop. 2.1], $C \cup D=$ $\mathbb{R}^{n}$ implies local existence of solutions and so the only way a maximal solution would not be complete is if it escapes to infinity during flows. From (18), the function $V$ is nonincreasing along all solutions to (16) so the $x_{p}$ component of each solution is bounded. Moreover, from continuity of $f_{u}$, boundedness of $x_{p}$ implies also boundedness of $\dot{u}$ along flows and thus forward completeness follows. Therefore, UGpAS implies UGAS of $\mathcal{A}$.

\section{PROOF OF THEOREMS 1 AND 2.}

The following lemma is needed to prove Theorems 1 and 2, and is similar to $[14$, Theorem 1,3$) \Rightarrow 1$ )].

Lemma 2: A closed set $\mathcal{A}$ is UGpAS for $\mathcal{H}:=(C, F, D, G)$ in (1) if it is UGS for $\mathcal{H}$ and, for each pair $\Delta>0, \delta>0$, it is $U G p A$ for $\mathcal{H}_{\delta, \Delta}$ in (3).

Proof. According to Definition 1, UGpAS of $\mathcal{A}$ follows from UGS (which is assumed) and UGpA, which is established next. With reference to Definition 1 , select any pair $r, \varepsilon$ of positive scalars and select $\Delta=\alpha(r)$ and $\delta=\alpha^{-1}(\varepsilon)$ (where $\alpha$ is the class $\mathcal{K}_{\infty}$ function establishing UGS of $\mathcal{H}$ ). By assumption, $\mathcal{H}_{\delta, \Delta}$ is UGpA, therefore there exists $T$ such that (2) holds for all solutions $x$ to $\mathcal{H}_{\delta, \Delta}$. For that same $T$, all solutions to $\mathcal{H}$ with $|x(0,0)|_{\mathcal{A}} \leq r$ satisfy (2) as well. Indeed, denote by $x$ any such solution and two cases may occur:

1) $|x(t, j)|_{\mathcal{A}}>\delta$ for all $(t, j) \in \operatorname{dom} x$, in which case notice that from UGS of $\mathcal{H}$ we have $|x(t, j)|_{\mathcal{A}} \leq \alpha(r)=\Delta$ for all $(t, j) \in \operatorname{dom} x$. Then, $x$ is also a solution to $\mathcal{H}_{\delta, \Delta}$ and (2) holds by definition.

2) there exists $(\bar{t}, \bar{j}) \in \operatorname{dom} x$ such that $|x(\bar{t}, \bar{j})|_{\mathcal{A}} \leq \delta$ and $|x(t, j)|_{\mathcal{A}}>\delta$ for all $(t, j) \in \operatorname{dom} x$ satisfying $t+j<\bar{t}+\bar{j}$. Then $x(t, j)$ is also a solution to $\mathcal{H}_{\delta, \Delta}$ for all $(t, j) \in \operatorname{dom} x$ with $t+j<\bar{t}+\bar{j}$ (so that it satisfies (2) by definition for all such times) while from UGS we have $|x(t, j)|_{\mathcal{A}} \leq \alpha(\delta)=\varepsilon$ for all $(t, j) \in \operatorname{dom} x$ with $t+j \geq \bar{t}+\bar{j}$ which implies (2) for the remaining part of $\operatorname{dom} x$.

Proof of Theorems 1 and 2. We only prove Theorem 1 as the proof of Theorem 2 is identical. Conditions (6) imply UGS of $\mathcal{H}$ following the same proof technique as in [4, Theo. 3.18].
Then using [4, Prop. 3.27] and Property 1 with Lemma 1, we conclude UGpA of $\mathcal{H}_{\delta, \Delta}$ for each pair of positive scalars $(\delta, \Delta)$. Finally the result follows from Lemma 2 .

\section{Proof of Claim 1}

To the end of proving Claim 1, we provide a robustness statement which strengthens the result in [1, Claim 5.1] (see also [13, Pages 332-333] for a similar result) establishing that under Assumption 2 the flow inequality (14) can be strengthened to hold robustly, as clarified below. The proof uses the regularity of $V_{p}$ and the continuity of $f_{p}$.

Lemma 3: Under Assumption 2, the following strengthened flow condition holds for all $x_{p} \in \mathbb{R}^{n_{p}}$

$$
\max _{\bar{f} \in \overline{\mathrm{co}}}\left\langle\nabla V_{p}\left(x_{p}, K\left(x_{p}\right), \bar{f}\right\rangle \leq-\rho_{\circ}\left(\left|x_{p}\right|_{\mathcal{A}_{p}}\right),\right.
$$

where $\overline{\mathrm{co}}$ denotes the closed convex hull.

Moreover, for each $\rho \in \mathcal{P} \mathcal{D}$ such that $\rho(s)<\rho_{\circ}(s), \forall s>0$, there exists a continuous function $\eta: \mathbb{R}^{n_{p}} \rightarrow[0, \infty)$ which is strictly positive on $\mathbb{R}^{n_{p}} \backslash \mathcal{A}_{p}$, such that for all $x_{p} \in \mathbb{R}^{n_{p}}$

$$
\left\langle\nabla V_{p}\left(x_{p}\right), \bar{f}\right\rangle \leq-\frac{\rho_{\circ}\left(\left|x_{p}\right| \mathcal{A}_{p}\right)+\rho\left(\left|x_{p}\right|_{\mathcal{A}_{p}}\right)}{2}, \forall \bar{f} \in F_{\eta}\left(x_{p}\right),
$$

where the set-valued mapping $F_{\eta}$ is defined as follows:

$$
F_{\eta}\left(x_{p}\right):=\overline{\mathrm{co}} f_{p}\left(x_{p}, K\left(x_{p}+\eta\left(x_{p}\right) \mathbb{B}\right)+\eta\left(x_{p}\right) \mathbb{B}\right) .
$$

Proof. Proof of (19). Fix $x_{p} \in \mathbb{R}^{n_{p}}$. By definition of the closed convex hull, for each $\bar{f} \in \overline{\operatorname{co}} f_{p}\left(x_{p}, K\left(x_{p}\right)\right)$, there exists a sequence $\left(f^{i}\right)_{i \in \mathbb{N}}$ in $\mathbb{R}^{n_{p}}$ such that $f^{i} \rightarrow \bar{f}$, as $i \rightarrow \infty$ satisfying, for each $i \geq 1$, the existence of two sequences $\left(\lambda^{i, j}\right)_{j=1, \ldots, i}$ in $[0,1]$ with $\sum_{j=1}^{i} \lambda^{i, j}=1$ and $\left(k^{i, j}\right)_{j=1, \ldots, i} \in$ $K\left(x_{p}\right)$ such that

$$
f^{i}=\sum_{j=1}^{i} \lambda^{i, j} f_{p}\left(x_{p}, k^{i, j}\right) .
$$

Now recalling (15), for each $i \in \mathbb{N}$ and for each $1 \leq j \leq i$, there exists a sequence $\left(x_{p}^{i, j, n}\right)_{n \in \mathbb{N}}$ such that

$$
\max \left\{\left|x_{p}-x_{p}^{i, j, n}\right|,\left|k^{i, j}-\kappa\left(x_{p}^{i, j, n}\right)\right|\right\} \leq \frac{1}{n}, \forall n \geq 1 .
$$

Then, using continuity of $\nabla V_{p}, f_{p}$ and $\rho_{\circ}$, and (14), we get

$$
\begin{aligned}
& \left.\left\langle\nabla V_{p}\left(x_{p}\right), \bar{f}\right\rangle=\lim _{i \rightarrow \infty} \sum_{j=1}^{i} \lambda^{i, j}\left\langle\nabla V_{p}\left(x_{p}\right), f_{p}\left(x_{p}, k^{i, j}\right)\right)\right\rangle \\
& =\lim _{i \rightarrow \infty} \sum_{j=1}^{i} \lambda^{i, j} \lim _{n \rightarrow \infty}\left\langle\nabla V_{p}\left(x_{p}^{i, j, n}\right), f_{p}\left(x_{p}^{i, j, n}, \kappa\left(x_{p}^{i, j, n}\right)\right)\right\rangle \\
& \leq-\lim _{i \rightarrow \infty} \sum_{j=1}^{i} \lambda^{i, j} \lim _{n \rightarrow \infty} \rho_{\circ}\left(\left|x_{p}^{i, j, n}\right|_{\mathcal{A}_{p}}\right) \\
& \leq-\lim _{i \rightarrow \infty} \sum_{j=1}^{i} \lambda^{i, j} \rho_{\circ}\left(\left|x_{p}\right|_{\mathcal{A}_{p}}\right) \leq-\rho_{\circ}\left(\left|x_{p}\right|_{\mathcal{A}_{p}}\right)
\end{aligned}
$$

where in the last line we used $\sum_{j=1}^{i} \lambda^{i, j}=1$.

Proof of (20). Using (19), the function $\eta$ is constructed following the proof technique of [1, Claim 5.1] (see also [13, Pages 332-333]), recognizing that in (20) we have 
$\frac{\rho_{\circ}\left(\left|x_{p}\right|_{\mathcal{A}_{p}}\right)+\rho\left(\left|x_{p}\right|_{\mathcal{A}_{p}}\right)}{2}<\rho_{\circ}\left(\left|x_{p}\right|_{\mathcal{A}_{p}}\right)$ for all $x_{p} \notin \mathcal{A}_{p}$ while in [1, Claim 5.1] we have $\frac{1}{2} V_{p}\left(x_{p}\right)<V_{p}\left(x_{p}\right), \forall x_{p} \notin \mathcal{A}_{p}$.

First select the function $\chi$ in (16a) as any continuous function satisfying $\chi\left(x_{p}\right)<\eta\left(x_{p}\right)$ for all $x_{p} \notin \mathcal{A}_{p}$, where $\eta$ is the continuous function introduced in Lemma 3. To prove the claim for hybrid system $\mathcal{H}$ with such a selection of $\chi$, fix scalars $\delta>0$ and $\Delta>0$ as in Property 1. Below we determine parameters $\gamma \in \mathcal{K}_{\infty}$ and $N>0$ satisfying (4) for all solutions $x$ to $\mathcal{H}_{\delta, \Delta}$. If the solution never jumps, then (4) trivially follows with any $N \geq 0$ and any class $\mathcal{K}_{\infty}$ function $\gamma$. If the solution jumps at least once, then by the restriction of the flow and jump sets of $\mathcal{H}_{\delta, \Delta}$, we have $|x(t, j)|_{\mathcal{A}}=\left|x_{p}(t, j)\right|_{\mathcal{A}_{p}} \leq \Delta$, for all $(t, j) \in \operatorname{dom} x$, $(t, j) \neq(0,0)$. Therefore, the (sub-)state $x_{p}$ is uniformly bounded as follows:

$$
\left|x_{p}(t, j)\right|_{\mathcal{A}_{p}} \leq \Delta, \quad \forall(t, j) \in \operatorname{dom} x, j \geq 1 .
$$

Since from (22) $x_{p}$ is bounded, then also $f_{u}\left(x_{p}\right)$ has a uniform upper bound and local boundedness of $K$ (inherited from $\kappa$ ) and continuity of $f_{p}$ and $f_{u}$ imply that there exist positive scalars $L_{x}$ and $L_{u}$, such that, for all $(t, j)$ in $\operatorname{dom} x, j \geq 1$,

$$
\begin{array}{r}
\left|x_{p}(t, j)-x_{p}\left(t_{j}, j\right)\right|<L_{x}\left(t-t_{j}\right) \\
\left|u(t, j)-u\left(t_{j}, j\right)\right|<L_{u}\left(t-t_{j}\right) .
\end{array}
$$

Since $\chi$ is continuous on $\mathbb{R}^{n_{p}}$, it is uniformly continuous on each compact set, namely there exists $L_{\chi}>0$ such that, for all $x_{p}^{i} \in \mathbb{R}^{n_{p}},\left|x_{p}^{i}\right|_{\mathcal{A}_{p}} \leq \Delta, i=1,2$,

$$
\left|\chi\left(x_{p}^{1}\right)-\chi\left(x_{p}^{2}\right)\right| \leq L_{\chi}\left|x_{p}^{1}-x_{p}^{2}\right| .
$$

Use now the result of Lemma 3 and, in particular, the continuous function $\eta$ satisfying (20) and the function $\chi$ in (16a) which is smaller than $\eta$ by construction. Select

$$
\begin{aligned}
\underline{\eta}_{\chi} & :=\frac{1}{2} \min _{\left|x_{p}\right| \mathcal{A}_{p} \in[\delta, \Delta]} \eta\left(x_{p}\right)-\chi\left(x_{p}\right) \\
\sigma_{\delta, \Delta} & :=\min \left\{\frac{\underline{\eta} \chi}{L_{x}}, \frac{\underline{\eta}_{\chi}}{L_{\chi} L_{x}}, \frac{\underline{\eta}_{\chi}}{L_{u}}\right\},
\end{aligned}
$$

and note that $\underline{\eta}_{\chi}>0$ (because $\chi\left(x_{p}\right)<\eta\left(x_{p}\right)$ for all $x_{p} \notin$ $\mathcal{A}_{p}$ ), implying $\sigma_{\delta, \Delta}>0$.

Then from (24) and the right bound of (27), we get for all $t \in\left[t_{j}, t_{j}+\sigma_{\delta, \Delta}\right]$

$$
f_{p}\left(x_{p}(t, j), u(t, j)\right) \in f_{p}\left(x_{p}(t, j), u\left(t_{j}, j\right)+\underline{\eta}_{\chi} \mathbb{B}\right) .
$$

Moreover, applying (26) first and then the left two bounds in (27) and (23), we get

$$
\begin{aligned}
\chi\left(x_{p}\right)+2 \underline{\eta}_{\chi} & \leq \eta\left(x_{p}\right) \\
x_{p}(t, j) & \in x_{p}\left(t_{j}, j\right)+\min \left\{\underline{\eta}_{\chi}, \frac{\underline{\eta}_{\chi}}{L_{\chi}}\right\},
\end{aligned}
$$

and the right bound of (29b) together with (25) gives

$$
\begin{aligned}
\chi\left(x_{p}\left(t_{j}, j\right)\right) & =\chi\left(x_{p}(t, j)\right)+\chi\left(x_{p}\left(t_{j}, j\right)\right)-\chi\left(x_{p}(t, j)\right) \\
& \subset \chi\left(x_{p}(t, j)\right)+L_{\chi}\left|x_{p}\left(t_{j}, j\right)-x_{p}(t, j)\right| \mathbb{B} \\
& \subset \chi\left(x_{p}(t, j)\right)+\underline{\eta}_{\chi} \mathbb{B} .
\end{aligned}
$$

Combining (29) with (28), we get the following relations where for compactness of notation we use $x_{p}$ for $x_{p}(t, j)$ and $\xi_{p}$ for $x_{p}\left(t_{j}, j\right)$ :

$$
\begin{aligned}
& f_{p}\left(x_{p}(t, j), u(t, j)\right) \\
\in & f_{p}\left(x_{p}, K\left(\xi_{p}+\chi\left(\xi_{p}\right) \mathbb{B}\right)+\chi\left(\xi_{p}\right) \mathbb{B}+\underline{\eta}_{\chi} \mathbb{B}\right) \\
\subset & f_{p}\left(x_{p}, K\left(x_{p}+\chi\left(x_{p}\right) \mathbb{B}+2 \underline{\eta}_{\chi} \mathbb{B}\right)+\chi\left(x_{p}\right) \mathbb{B}+2 \underline{\eta}_{\chi} \mathbb{B}\right) \\
\subset & f_{p}\left(x_{p}, K\left(x_{p}+\eta\left(x_{p}\right) \mathbb{B}\right)+\eta\left(x_{p}\right) \mathbb{B}\right)=F_{\eta}\left(x_{p}(t, j)\right),
\end{aligned}
$$

for all $t \in\left[t_{j}, t_{j}+\sigma_{\delta, \Delta}\right]$. Then from (20) we obtain

$$
\begin{aligned}
& \left\langle\nabla V_{p}(x(t, j)), f_{p}(x(t, j), u(t, j))\right\rangle \\
& \quad \leq-\frac{1}{2}\left(\rho_{\circ}\left(\left|x_{p}(t, j)\right|_{\mathcal{A}_{p}}\right)+\rho\left(\left|x_{p}(t, j)\right|_{\mathcal{A}_{p}}\right)\right) \\
& \quad<-\rho\left(|x(t, j)|_{\mathcal{A}}\right) .
\end{aligned}
$$

Combined with (16b) this latter inequality implies that the solution is not in the jump set for all $t \in\left[t_{j}, t_{j}+\sigma_{\delta, \Delta}\right]$. This dwell time for all $j \geq 1$, suggests the selection of $\gamma(s)=$ $\sigma_{\delta, \Delta} s$ that, combined with the selection $N=\sigma_{\delta, \Delta}$ (ensuring that the right hand side of (4) is non-positive for $(t, j) \in$ dom $x, j=0$ ), implies (4). (Note that establishing condition (8) would require additional steps in this proof. This shows that (4) is a simpler condition to check for this example.)

\section{REFERENCES}

[1] C. Cai, A.R. Teel, and R. Goebel. Smooth Lyapunov functions for hybrid systems - Part I: existence is equivalent to robustness. IEEE Transactions on Automatic Control, 52(7):1264-1277, 2007.

[2] Dimos V Dimarogonas, Emilio Frazzoli, and Karl H Johansson. Distributed event-triggered control for multi-agent systems. IEEE Transactions on Automatic Control, 57(5):1291-1297, 2012.

[3] MCF Donkers and WPMH Heemels. Output-based event-triggered control with guaranteed $L_{\infty}$-gain and improved and decentralized eventtriggering. IEEE Transactions on Automatic Control, 57(6):1362-1376, 2012 .

[4] R. Goebel, R. G. Sanfelice, and A. R. Teel. Hybrid Dynamical Systems. Princeton University Press, 2012.

[5] R. Goebel, R.G. Sanfelice, and A.R. Teel. Hybrid dynamical systems. IEEE Control Systems Magazine, 29(2):28-93, 2009.

[6] R. Goebel and A. R. Teel. Solutions to hybrid inclusions via set and graphical convergence with stability theory applications. Automatica, 42(4):573-587, 2006

[7] R. Goebel and A. R. Teel. Preasymptotic stability and homogeneous approximations of hybrid dynamical systems. SIAM Review, 52(1):87109, 2010.

[8] C. Prieur, S. Tarbouriech, and L. Zaccarian. Lyapunov-based hybrid loops for stability and performance of continuous-time control systems. Automatica, 49(2):577-584, 2013.

[9] R.G. Sanfelice, R. Goebel, and A. R. Teel. Invariance principles for hybrid systems with connections to detectability and asymptotic stability. IEEE Transactions on Automatic Control, 52(12):2282-2297, 2007.

[10] R.G. Sanfelice and A. R. Teel. Asymptotic stability in hybrid systems via nested Matrosov functions. IEEE Transactions on Automatic Control, 54(7):1569-1574, 2009.

[11] A. Seuret, C. Prieur, and N. Marchand. Stability of non-linear systems by means of event-triggered sampling algorithms. IMA Journal of Mathematical Control and Information, to appear, 2013.

[12] Paulo Tabuada. Event-triggered real-time scheduling of stabilizing control tasks. IEEE Transactions on Automatic Control, 52(9):1680$1685,2007$.

[13] A. R. Teel and L. Praly. A smooth Lyapunov function from a class- $\mathcal{K} \mathcal{L}$ estimate involving two positive semidefinite functions. ESAIM Control Optim. Calc. Var., 5:313-367, 2000.

[14] A.R. Teel, E. Panteley, and A. Loria. Integral characterizations of uniform asymptotic and exponential stability with applications. Math. Contr. Sign. Syst., 15:177-201, 2002. 